\title{
Peritonite Meconial como Diagnóstico Diferencial de Ascite Fetal: Relato de Caso
}

\author{
Meconium Peritonitis in the Differential Diagnosis of Fetal Ascites: a Case Report
}

\author{
Melania Maria Ramos de Amorim, Paulo Carvalho Vilela, Luiz Carlos Santos \\ Gilliatt Hanois Falbo Neto, Ozanil Cursino, Fernando Amaral, \\ Karina Maria Azevedo Bitencourt
}

\section{RESUMO}

Introdução: a peritonite meconial, como resultado da perfuração intestinal fetal, apresenta baixa incidência (1:30.000 nascimentos) e elevada mortalidade (em torno de 50\%). Os achados ecográficos pré-natais incluem ascite e calcificações intra-abdominais. Há evidências de que o diagnóstico pré-natal possa melhorar o prognóstico pós-natal.

Relato do Caso: R.C.M.S., 22 anos, II gesta O para, realizou ultra-sonografia em 02/12/98 com diagnóstico de ascite fetal. Fez investigação para hidropisia fetal, afastando-se causas imunes e não-imunes. Foram realizados ecografias seriadas em que se manteve a imagem de ascite fetal acentuada, sem calcificações. Parto normal em 02/01/99, com 36 semanas, observando-se volumoso poliidrâmnio. Recém-nascido do sexo feminino pesando 2.670 gramas, com sinais de desconforto respiratório, abdome distendido e com petéquias. Apresentou aumento progressivo da distensão abdominal, palpação de massa pétrea no hipocôndrio direito e eliminação de muco branco ao toque retal. Raios-x em 04/01/99 com imagem de extensas calcificações abdominais, distensão de alças intestinais e ausência de gás na ampola retal. Hipótese diagnóstica de peritonite meconial. Indicada laparotomia exploradora em 04/01/99, encontrando-se volumoso cisto meconial e atresia ileal, realizandose lise de aderências e ileostomia em dupla boca. Evolução satisfatória nos primeiros dias de pós-operatório, complicada posteriormente por quadro séptico, verificando-se o óbito neonatal em 09/01/99.

Conclusão: a peritonite meconial deve ser lembrada no diagnóstico diferencial das causas de ascite fetal. O diagnóstico pré-natal no presente caso poderia ter antecipado a indicação cirúrgica, com possivel melhora da evolução neonatal.

PALAVRAS-CHAVE: Peritonite meconial. Ascite fetal. Diagnóstico pré-natal.

\section{Introdução}

A peritonite meconial constitui uma reação exsudativa inflamatória peritoneal de origem química, secundária a uma perfuração intestinal, sendo o íleo o local mais freqüente ${ }^{3,7,10,18,21}$. Incide em 1 de cada 30.000-35.000 nascimentos ${ }^{10}$,

\footnotetext{
Instituto Materno-Infantil de Pernambuco (IMIP)

Correspondência:

Melania Maria Ramos de Amorim

Coordenação de Ensino do IMIP

Rua dos Coelhos, 300

50.070-550 - Recife - PE

e-mail: andfpc@br.homeshopping.com.br
}

descrevendo-se, em geral, uma relação sexo masculino/feminino de $1,5 / 1^{9}$. Em sua etiopatogênese estão envolvidos diversos fatores, como a obstrução orgânica (volvo, atresia ou íleo meconial), patologia vascular e funcional ${ }^{10}$ ou etiologia viral (hepatite, varicela, citomegalovírus, parvovírus) ${ }^{13,16}$. A mucoviscidose pode ser encontrada em $10 \%$ dos casos, principalmente quando não há calcificações abdominais ${ }^{7,10,15}$. A peritonite meconial pode também estar associada à doença de Hirschsprung ${ }^{9}$ e doença autossômica recessiva ${ }^{5}$; metade dos casos de Gaillard et al. ${ }^{8}$ estavam associados à trissomia do $21^{21}$.

Nos últimos anos o diagnóstico pré-natal da 
peritonite meconial tem sido mais comum, devido à presença de achados ultrassonográficos de polidrâmnio, ascite fetal, dilatação de alças intestinais e calcificações ${ }^{6,7,10,12,19}$. Estes achados, de acordo com Hsu (1990), são encontrados em respectivamente $30 \%, 100 \%$, 99\% e $35 \%$ dos casos $^{9}$. O diagnóstico diferencial deve ser feito com hidropisia fetal, linfangioma cístico, ascite urinosa, teratoma e tumores vasculares ${ }^{6,10}$. A decisão do momento do parto depende da evolução ultrassonográfica e a via de parto tem indicação obstétrica ${ }^{10}$. Segundo Shyu et al. ${ }^{17}$, pode-se realizar paracentese para diminuir a pressão intra-torácica, irritação peritoneal e a distocia durante o trabalho de parto.

Atualmente, a sobrevida não ultrapassa 50\%, mesmo com o diagnóstico realizado durante o prénatal $^{6,10,18}$. Wang ${ }^{22}$ relata em seu estudo que a mortalidade foi maior quando o diagnóstico foi realizado no período pós-natal.

No presente estudo, os autores relatam um caso de peritonite meconial diagnosticado antes do nascimento como hidropisia fetal. Julgamos relevante sua apresentação devido não apenas à raridade mas também à importância de se considerar esta condição no diagnóstico diferencial de ascite fetal determinada ecograficamente.

\section{Relato do Caso}

R.C.M.S., 22 anos, II gesta O para (1 abortamento provocado durante o $4^{\circ}$ mês), foi admitida na Enfermaria de Alto Risco do IMIP em 2/12/98, com idade gestacional de 31/32 semanas (ultra-sonografia corrigida de primeiro trimestre). Trazia ultra-sonografia realizada durante o prénatal (2/12/1998) que evidenciava líquido amniótico aumentado $(+/++)$ e ascite fetal de moderada intensidade, sem outras malformações fetais. Tinha iniciado acompanhamento pré-natal no segundo mês de gestação, no IMIP, tendo feito 5 consultas. Recebeu vacina antitetânica. Negava manobras abortivas, contato com doenças infecciosas, uso de drogas, exposição a radiação e quaisquer outras intercorrências. Negava antecedentes de malformações na família.

O exame físico geral não detectou anormalidades, verficando-se uma pressão arterial de 110 x $70 \mathrm{mmHg}$. Ao exame obstétrico observamos, útero de tono normal e sem dinâmica; altura de fundo uterino de $37 \mathrm{~cm}$. Feto em situação longitudinal, apresentação cefálica. Batimentos cardiacos fetais $(\mathrm{BCF})=140 \mathrm{bpm}$. Toque: colo apagado $20 \%$, pérvio para $2 \mathrm{~cm}$, apresentação cefálica, bolsa integra.
As hipóteses diagnósticas no internamento foram de hidropisia fetal não-imune e poliidrâmnio, interrogando-se malformação fetal.

Os exames complementares realizados entre 2 e 10/12/1998 apresentaram os seguintes resultados: Classificação sangüinea: A positivo; VDRL negativo; curva glicêmica: normal; toxoplasmose: IgG e IgM não-reagentes; rubéola: IgG reagente, IgM não-reagente; citomegalovirus: IgG e IgM não-reagentes.

A ultra-sonografia de 2/12/1998 mostrou feto único em situação longitudinal, com apresentação cefálica e dorso à esquerda. BCF presentes e rítmicos. Diâmetro biparietal $=7,8 \mathrm{~cm}$; circunferência abdominal $(\mathrm{CA})=30,7 \mathrm{~cm}$; comprimento do fêmur $(\mathrm{CF})=5,6 \mathrm{~cm}$. Perfil biofisico fetal normal. Placenta grau zero. Índice do líquido amniótico de $22 \mathrm{~cm}$. A idade gestacional foi avaliada em 31/32 semanas. Ascite fetal de moderada a grande intensidade. Não foram identificadas quaisquer outras alterações na morfologia fetal. Relação CF/CA de 18,6. Realizou-se ecocardiografia fetal, sem anormalidades.

Como não foram encontradas causas aparentes da ascite fetal, afastadas as principais etiologias, a paciente teve alta hospitalar em 10/2/1998, com hipótese diagnóstica de hidropisia fetal não-imune, recebendo orientação de controle ecográfico ambulatorial. Administrou-se corticoterapia para aceleração da maturidade pulmonar fetal (betametasona 12 mg IM - 31/12/98 e 1/1/ 99).

A paciente submeteu-se a mais 2 ultrasonografias em 10/12/98 e 29/12/98, mantendose o achado ultra-sonográfico de ascite fetal, sem outras anormalidades. Foi admitida no Serviço em trabalho de parto no dia $2 / 1 / 99$, evoluindo para parto transpelvino às $7 \mathrm{~h} 30 \mathrm{~min}$ do dia $3 / 1 / 99$. À amniotomia, observou-se saída de abundante quantidade de líquido amniótico citrino, com grumos (poliidrâmnio).

O recém-nascido era do sexo feminino, tendo pesado 2.670 gramas. Apresentou escores de Apgar de 4 e 7 no $1^{\circ}$ e $5^{\circ}$ minutos. Necessitou de estimulação tátil e ventilação com pressão positiva. A idade gestacional estimada pelo Capurro somático foi de 36 semanas e 1 dia. Ao exame após o nascimento, apresentava aspecto geral regular, encontrando-se dispnéico $(++/ 4+)$, gemente, cianótico, apático, com batimentos de asa de nariz. Ausculta respiratória: murmúrio vesicular (MV) rude, sem ruídos adventícios. Abdome distendido, com presença de edema, petéquias e equimose. Passada sonda nasogástrica. Ânus pérvio.

A placenta era de aspecto macroscópico normal, com edema discreto e algumas calcificações. 
As hipóteses diagnósticas no nascimento foram: 1) Recém-nascido pré-termo adequado para a idade gestacional; 2) hipóxia moderada; 3) desconforto respiratório: taquipnéia transitória do RN?; 4) infecção congênita?

O RN evoluiu com piora do quadro, agravando-se o desconforto respiratório. Foi então submetido à ventilação mecânica assistida (VMA). Não houve eliminação de mecônio. Iniciou tratamento com ampicilina e gentamicina EV em 3/1/99 (leucograma infeccioso). No dia 04/01/99 manifestou-se piora da distensão abdominal, sendo então o RN avaliado pela Cirurgia Pediátrica. Ao exame físico, evidenciavam-se, além de distensão abdominal, equimoses e petéquias, massa pétrea no hipocôndrio direito. Ao toque retal, verificou-se eliminação de muco branco (Figura 1). Realizaramse ultra-sonografia e raios-x de tórax e abdome (ver abaixo). Aventada a hipótese diagnóstica de peritonite meconial, sendo indicada laparotomia. Recebeu hemotransfusão, com dois concentrados de hemácias e duas unidades de plasma.

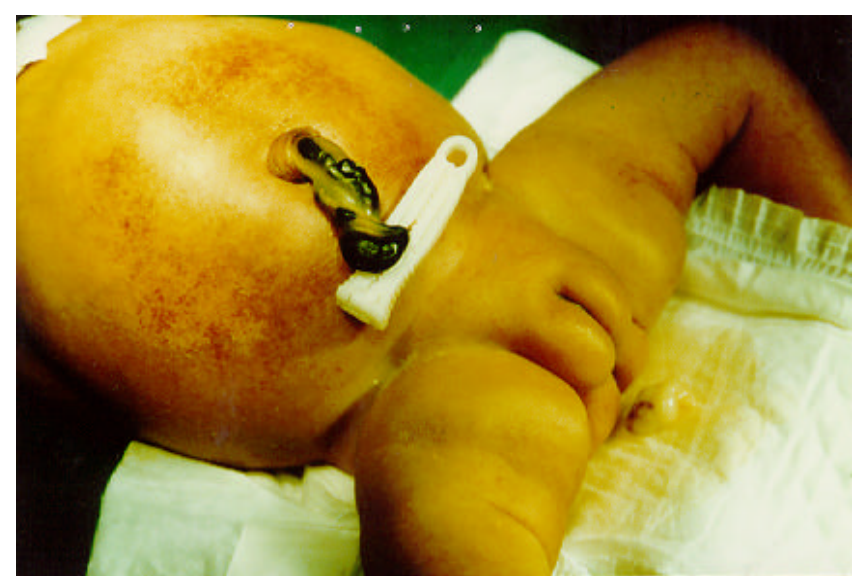

Figura 1 - Recem-nascido com abdome distendido, edema, equimose e eliminação de muco branco

Os resultados dos exames laboratoriais são apresentados a seguir: Leucograma - o exame de $3 / 1 / 99$ apresentava 23.882 leucócitos $/ \mathrm{mm}^{3}$, com contagem diferencial de 5 pró-mielócitos, 1 mielócito, 3 metamielócitos, 28 bastões, 24 segmentados. No dia 4/1/99, encontraram-se 35.000 leucócitos $/ \mathrm{mm}^{3}$, 1 mielócito, 2 metamielócitos, 12 bastões e 54 segmentados. Hemoglobina de 8,2 g\% em 4/1/99.

Radiografia de abdome (em 4/1/99): extensas calcificações em placa distribuídas no abdome, com predominio no quadrante superior direito, compativeis com peritonite meconial calcificada. Ausência de gás na ampola retal e presença de moderada dilatação das alças de intestino delgado até a região do íleo terminal. Hipótese diagnóstica: atresia ileal - íleo meconial (Figura 2).

A ultra-sonografia abdominal em 4/1/99 mostrou figado e vias biliares normais, observando- se material ecogênico com sombra acústica posterior, correspondendo às calcificações (peritonite meconial?) já descritas em radiografia simples de abdome. Ausência de coleções intracavitárias significativas.

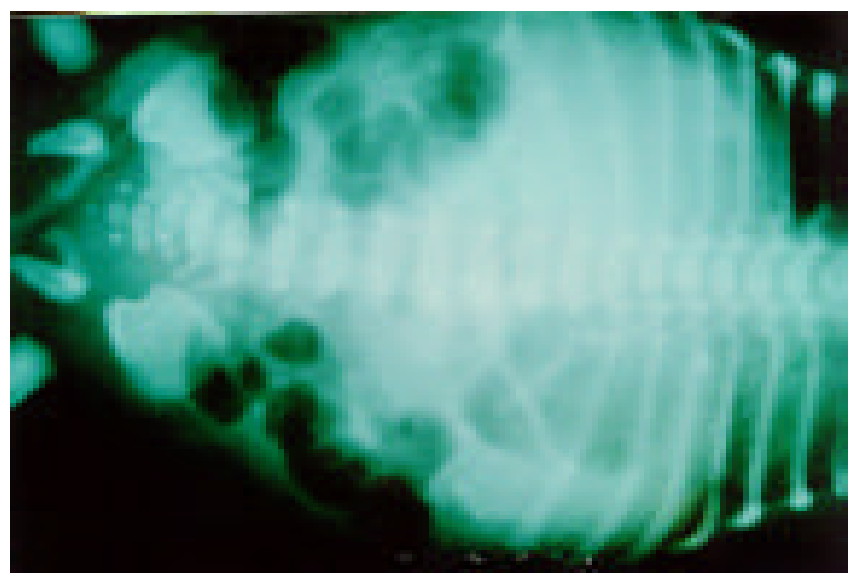

Figura 2 - Radiografia de abdome, extensas calcificações e dilatação de intestino delgado

Realizou-se laparotomia exploradora em 4/1/ 99. Após incisão transversa no hipocôndrio direito, encontraram-se os seguintes achados cirúrgicos: peritônio espessado, com aumento da vascularização e coloração esverdeada. Não foi possivel penetrar a cavidade sem passar através da coleção de mecônio, que ocupava todo o hipocôndrio direito (Figura 3). Havia aderências firmes entre as alças e uma dilatação importante das alças do íleo terminal, o qual terminava conectado à membrana que envolvia o bolsão de mecônio. Aderido à parede do bolsão de mecônio encontrava-se o segmento distal do íleo terminal medindo aproximadamente $4 \mathrm{~cm} \mathrm{e}$ continuando-se com a válvula ileo-cecal e colons os quais encontravam-se com aspecto de desuso (microcólon). O intestino delgado media cerca de 40 $\mathrm{cm}$ do ângulo de Treitz até a sua ligação com o bolsão de mecônio, parecendo encurtado em sua extensão. Realizou-se então lise de aderências e ileostomia em dupla boca, firmando-se o diagnóstico pós-operatório de peritonite meconial e atresia de íleo.

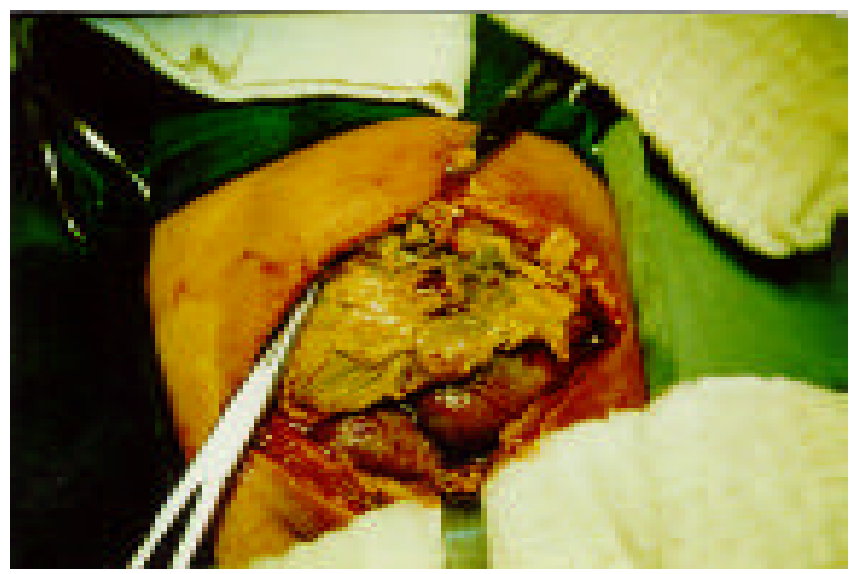

Figura 3 - Coleção de mecônio que ocupava todo o hipocôndrio direito 
No pós-operatório, o RN evoluiu inicialmente com melhora clínica, sendo retirado do respirador, passando-se para CPAP nasal. A antibioticoterapia foi mantida. Recebeu nova hemotransfusão com 1 concentrado de hemácias, em 6/1/99. Houve redução progressiva da FiO2. O abdome era depressivel, com edema de parede, e a ileostomia funcionava satisfatoriamente. Foi solicitada avaliação para fibrose cística do pâncreas. Em 8/1/99 apresentou piora do estado geral, encontrando-se taquipnéico, febril, hipocorado, desidratado, com fácies de sofrimento. Os pulmões estavam hipoventilados, com estertores, verificandose tiragem intercostal, freqüência cardiaca de 198 bpm e freqüência respiratória de $72 \mathrm{bpm}$. Reiniciada ventilação mecânica assistida em 8/1/99. Em 9/1/99 apresentou parada cardiorrespiratória irreversivel, sendo constatado o óbito às 6 h30 min.

\section{Discussão}

A peritonite meconial é uma entidade rara, porém nos últimos anos vem se observando um aumento de sua incidência, com maior freqüência de diagnóstico pré e pós-natal. Relatos de casos diversos têm sido publicados na literatura mundial ${ }^{1,4,14}$. Conquanto o prognóstico persista desfavorável, alguns autores aventam a possibilidade de que o diagnóstico pré-natal possa melhorá- $10^{6,22}$.

De acordo com Dirkes et al. ${ }^{6}$, apenas $22 \%$ dos fetos com um diagnóstico pré-natal de peritonite meconial desenvolvem complicações requerendo cirurgia pós-natal. Na ausência de malformações intestinais (peritonite meconial "simples"), a regressão espontânea intra-útero já foi descrita ${ }^{7}$. No entanto, a associação com malformações, em geral a atresia ileal, descrita como peritonite meconial "complexa" determina piora do prognóstico.

No caso que apresentamos, a peritonite meconial encontrava-se associada a atresia ileal, com achado radiológico característico de calcificações intra-abdominais que, no entanto, não foram evidenciadas pela ultra-sonografia pré-natal. O achado ecográfico de ascite fetal levou à hipótese diagnóstica de hidropisia fetal não-imune, não tendo sido aventada a possibilidade de peritonite meconial. Há referências na literatura da peritonite meconial como diagnóstico diferencial de hidropisia não-imune ${ }^{3}$, uma vez que a ascite constitui um dos achados mais freqüentes e nem sempre as calcificações são evidentes pela ultra-sonografia.

Acreditamos que a falta de diagnóstico prénatal tenha complicado a evolução neonatal no presente caso, uma vez que houve retardo no diagnóstico pós-natal, tendo sido realizada intervenção cirúrgica tardiamente, com o recém- nascido em condições desfavoráveis. O óbito, já no $4^{\circ}$ dia pós-operatório, depois de uma melhora clínica inicial, associou-se com o quadro de sepse secundário à infecção hospitalar, talvez evitável se a cirurgia tivesse sido indicada precocemente. Vale ressaltar, todavia, que mesmo quando se estabelece o diagnóstico pré-natal, a mortalidade é ainda elevada quando existem malformações intestinais ${ }^{11}$.

Taxas elevadas de mortalidade, em torno de $40-60 \%$, têm sido descritas para estes $\operatorname{casos}^{2,18,20}$, em contraste a taxas mais baixas, em torno de $10 \%$, para a peritonite meconial simples com diagnóstico pré-natal 3, 6. Wang et al. ${ }^{22}$ (1994), no entanto, descreveram uma baixa taxa de mortalidade (1 em 10 casos) mesmo para a peritonite meconial complexa, associada a atresia ou perfuração ileal. Os autores creditam esse resultado favorável em parte ao diagnóstico pré-natal (realizado com sucesso em 4 pacientes) e, em parte, ao progresso nos cuidados intensivos no periodo pré-natal. Melhores resultados nesta doença potencialmente fatal podem portanto ser antecipados pela cooperação entre neonatologistas, cirurgiões pediátricos e obstetras ${ }^{22}$.

\section{SUMMARY}

Introduction: meconium peritonitis as result of fetal intestinal perforation has a low incidence (1:30,000 deliveries) and high mortality (50\% or more). Prenatal ultrasound findings include fetal ascites and intra-abdominal calcifications. Evidence suggests that prenatal diagnosis can improve postnatal prognosis.

Case Report: R.C.M.S., 22 years, II pregnancy O para, presented ultrasound (12/02/98) with diagnosis of fetal ascites. Investigation for hydrops fetalis was performed and immune and nonimmune causes were excluded. Severe fetal ascites persisted on subsequent ultrasound examinations, without calcifications. Vaginal delivery occurred at 36 weeks (01/02/99), with polyhydramnios. Female neonate weighing 2,670 g, with signs of respiratory distress, abdominal distension and petechiae. Abdominal distension worsened progressively, with palpation of a petrous tumor in the right upper quadrant and elimination of white mucus at rectal examination. Radiological findings $(01 / 04 / 99)$ were disseminated abdominal calcifications, intestinal dilatation and absence of gas at rectal ampulla. Exploratory laparotomy was indicated with diagnosis of meconium peritonitis. A giant meconium cyst and ileal atresia were observed and lysis of adhesions and ileostomy were performed. Initial postoperative evolution was satisfactory but was subsequently complicated by sepsis and neonatal death occurred (01/09/99).

Conclusion: meconium peritonitis should be remembered at differential diagnosis of fetal ascites. In the present case, surgical indication could be anticipated if prenatal diagnosis were established, with improvement of neonatal evolution.

KEY WORDS: Meconium peritonitis. Ascites, fetal. Prenatal diagnosis. 


\section{Referências}

1. Akindele JA. Intestinal bilious vomiting - an unusual presentation of intestinal atresia in the newborn. Afr J Med Sci 1994; 23: 193-4.

2. Brugman SM, Bjelland JJ, Thomassin JE. Sonographic findings with radiologic correlation in meconium peritonitis. J Clin Ultrasound 1979: 7: 305-6.

3. Chalubinski K, Deutinger J, Bernaschek G. Meconium peritonitis: extrusion of meconium and different sonographical appearances in relation to the stage of the disease. Prenat Diagn 1992; 12: 631-6.

4. Chiba T, Ohi R, Kamiyama T, Yoshida S. Ileal atresia with perforation in siblings. Eur J Pediatr Surg 1991; 1: 51-3.

5. Chitayat D, Grisaru S, Ryan G, Toi A, Filler R, Seaward GR et al. Familial ileal perforation: prenatal diagnosis and postnatal follow-up. Prenat Diagn 1998; 18: 78-82.

6. Dirkes K, Crombleholme TM, Craigo SD, Latchaw LA, Jacir NN, Harris BH, DAlton ME The natural history of meconium peritonitis diagnosed in utero. J Pediatr Surg 1995; 30: 979-82.

7. Estroff JA, Bromley B, Benacerraf BR. Fetal meconium peritonitis without sequelae. Pediatr Radiol 1992; 22: $277-8$.

8. Gaillard D, Bouvier R, Scheiner C, Nessmann C, Delezoide AL, Dechelotte P, Leheup B, Cordier MP, Carles D, Lallemand A. Meconium ileus and intestinal atresia in fetuses and neonates. Pediatr Pathol Lab Med 1996; 16: 25-40.

9. Hsu CH, Jen LW, Huang FY, Chen MR, Kao HA, Ho WY, Yeh ML, Chang PY. Clinical observation of meconium peritonitis. Acta Paediatr Sin 1990; 31: 214-20.

10. Isfer E, Sanchez RC, Saito M. Medicina Fetal: Diagnóstico e Conduta. Editora Revinter, Rio de Janeiro, 1996.p.138-40.

11. Konje JC, de Chazal R, MacFadyen U, Taylor DJ Antenatal diagnosis and management of meconium peritonitis: a case report and review of the literature. Ultrasound Obstet Gynecol 1995; 6: 66-9.
12. Lin $\mathrm{MH}$, Jeng CJ, Wang KG, Yang YC. Prenatal diagnosis of meconium peritonitis-a case report with literature review. Chung Hua I Hsueh Tsa Chih 1992; 49: 48-52.

13. Pletcher BA, Williams MK, Mulivor RA, Barth D, Linder C, Rawlinson K. Intrauterine cytomegalovirus infection presenting as fetal meconium peritonitis. Obstet Gynecol 1991; 78: 903-5.

14. Raman S, Chan LL, Chang KW, Rachagan SP. Prenatal diagnosis of intestinal obstruction due to ileal atresia. Med J Malaysia 1992; 47: 22830 .

15. Rescorla FJ, Grosfeld JL. Contemporary management of meconium ileus. World J Surg 1993; 17: 318-25.

16. Schild RL, Plath H, Thomas P, Schulte Wissermann $\mathrm{H}$, Eis Hübinger AM, Hansmann M Fetal parvovirus B19 infection and meconium peritonitis. Fetal Diagn Ther 1998; 13: $15-8$.

17. Shyu MK, Chen CD, Hsieh FJ, Yau KI, Lin GJ, Lee ML. Intrauterine intervention in a case of recurrent meconium peritonitis. Prenat Diagn 1994; 14: 993-5.

18. Soong JH, Hsieh CC, Chiu TH, Kuo DM, Chen FP, Lo LM, Soong YK. Meconium peritonitis-antenatal diagnosis by ultrasound. Chang Keng I Hsueh 1992; 15: 155-60.

19. Sukcharoen N. Prenatal sonographic diagnosis of meconium peritonitis: a case report. J Med Assoc Thai 1993; 76: 171-6.

20. Tibboel D, Gaillard JLJ, Molenaar JC. The importance of mesenteric vascular insufficiency in meconium peritonitis. Hum Pathol 1996 17: 411-6.

21. Tseng CW, Chao AS, Chang FH, Chang SY, Soong YK. Color Doppler energy in prenatal diagnosis of meconium peritonitis: a case report. Chang Keng I Hsueh 1997; 20: 58-61.

22. Wang YJ, Chen HC, Chi CS. Meconium peritonitis in neonates. Chung Hua I Hsueh Tsa Chih 1994; 53: 49-53. 